\title{
BMJ Open \\ Mental health and functional impairment outcomes following a 6-week intensive treatment programme for UK military veterans with post-traumatic stress disorder (PTSD): a naturalistic study to explore dropout and health outcomes at follow-up
}

\author{
Dominic Murphy, ${ }^{1,2}$ Georgina Hodgman, ${ }^{1}$ Carron Carson, ${ }^{1}$ Lucy Spencer-Harper, ${ }^{1}$ \\ Mark Hinton, ${ }^{1}$ Simon Wessely, ${ }^{2}$ Walter Busuttil ${ }^{1}$
}

To cite: Murphy $D$,

Hodgman G, Carson C, et al. Mental health and functional impairment outcomes following a 6-week intensive treatment programme for UK military veterans with post-traumatic stress disorder (PTSD): a naturalistic study to explore dropout and health outcomes at follow-up. BMJ Open 2015;5:e007051. doi:10.1136/bmjopen-2014007051

- Prepublication history for this paper is available online. To view these files please visit the journal online (http://dx.doi.org/10.1136/ bmjopen-2014-007051).

Received 30 October 2014 Revised 27 February 2015 Accepted 2 March 2015

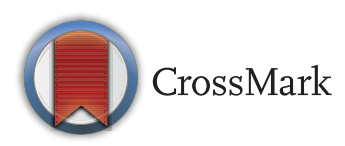

${ }^{1}$ Combat Stress, Leatherhead, UK

${ }^{2}$ King's Centre for Military Health Research, King's College London, London, UK

Correspondence to Dr Dominic Murphy; dominic. murphy@combatstress.org.uk

\section{ABSTRACT}

Objective: Combat Stress, a UK national charity for veterans with mental health problems, has been funded by the National Health Service (NHS) to provide a national specialist service to deliver treatment for posttraumatic stress disorder (PTSD). This paper reports the efficacy of a PTSD treatment programme for UK veterans at 6 months follow-up.

Design: A within subject design.

Setting: UK veterans with a diagnosis of PTSD who accessed Combat Stress.

Participants: 246 veterans who received treatment between late 2012 and early 2014.

Intervention: An intensive 6-week residential treatment programme, consisting of a mixture of individual and group sessions. Participants were offered a minimum of 15 individual trauma-focused cognitive behavioural therapy sessions. In addition, participants were offered 55 group sessions focusing on psychoeducational material and emotional regulation.

Main outcome measures: Clinicians completed measures of PTSD and functional impairment and participants completed measures of PTSD, depression, anger and functional impairment.

Results: We observed significant reductions in PTSD scores following treatment on both clinician completed measures (PSS-I: $-13.0,95 \% \mathrm{Cl}-14.5$ to -11.5 ) and self-reported measures (Revised Impact of Events Scale (IES-R): $-16.5,95 \% \mathrm{Cl}-19.0$ to -14.0$)$.

Significant improvements in functional impairment were also observed (eg, Health of the Nation Outcome Scales (HONOS): $-6.85,95 \% \mathrm{Cl}-7.98$ to -5.72 ). There were no differences in baseline outcomes between those who completed and those who did not complete the programme, or post-treatment outcomes between those we were able to follow-up at 6 months and those lost to follow-up.

\section{Strengths and limitations of this study}

- First study of its kind in the UK to evaluate outcomes following treatment in veterans with posttraumatic stress disorder (PTSD).

- The treatment offered has been standardised to increase treatment fidelity.

- The study sampled from a national treatment programme offered by the largest provider of interventions for veterans with PTSD in the UK.

- Over $80 \%$ of our sample was followed up 6 months later to explore treatment outcomes.

- A non-responder study was completed that found no evidence of a response bias.

- The study did not employ an RCT design so there are limitations about the conclusions that can be drawn.

- Little was known about the treatment experiences of participants prior to them enrolling for treatment.

Conclusions: In a naturalistic study we observed a significant reduction in PTSD scores and functional impairment following treatment. These improvements were maintained at 6 month follow-up. Our findings suggest it may be helpful to take a closer look at combining individual trauma-focused cognitive behaviour therapy and group sessions when treating veterans with PTSD. This is the first UK study of its kind, but requires further evaluation.

\section{INTRODUCTION}

Since the start of the UK military interventions in Iraq and Afghanistan there has been considerable public, political and media concerns about the treatment of serving and 
ex-serving members of the UK military who have served in either or both conflicts. There is robust epidemiological evidence to suggest prevalence rates of posttraumatic stress disorder (PTSD) within veterans from these deployments to be around $4 \%$, with increased rates in reservists and those in combat roles. ${ }^{1}$ While there are existing guidelines for the treatment of PTSD, ${ }^{2}$ there have been no studies to our knowledge of treatment outcomes within UK ex-serving personnel; with most of the evidence coming from studies conducted in the USA, Australia, Canada and Israel. ${ }^{3-8}$ Differences in prevalence, culture and healthcare systems mean that caution should be observed before assuming that data from other countries can be applied to the UK situation.

It has been observed that military personnel with PTSD struggle to engage in help-seeking behaviours ${ }^{9} 10$ and meta analyses have shown that interventions for veterans with PTSD are less effective than for other populations with PTSD. ${ }^{11}{ }^{12}$ To explore how to improve engagement with clinical services in veteran populations the UK Ministry of Defence funded a pilot initiative between 2007 and 2010 to provide six dedicated veterans clinics. Evaluation of this pilot demonstrated that veterans were more likely to attend and engage in treatment compared to traditional National Health Service (NHS) mental health services. ${ }^{13}$ What seemed vital was to provide services in a veteran dedicated clinic.

Combat Stress is the largest and most significant provider of mental health services for veterans in the UK and inpatient psychological treatment; and runs national community and outreach services. In 2011, Combat Stress was awarded NHS funding to provide a national specialist service for veterans with PTSD, and developed the first systematic and evaluated programme to treat UK veterans with PTSD. This is a 6-week treatment programme which is underpinned by cognitive behavioural therapy (CBT) and the provision of individual trauma-focused CBT (TF-CBT). This is supported by meta-analytic reviews that demonstrate the efficacy of TF-CBT for the treatment of chronic PTSD. ${ }^{11}{ }^{14}$ Combat Stress was funded to provide services to a population of veterans who were deemed to be treatment resistant because they had failed to engage with mainstream NHS services.

In this paper we report the efficacy of the 6 weeks treatment programme for individuals with chronic PTSD across a range of outcomes at a 6-month follow-up period. Outcomes included measures of PTSD, comorbid psychological difficulties and functioning. For this study chronic presentation of PTSD has been defined as being exposed to two or more traumatic events, the presence of comorbid mental health difficulties and evidence of functional impairment.

\section{METHODS}

Setting

In the UK a veteran is defined as an individual who has completed at least 1 day of military service and is no longer employed by the Armed Forces. ${ }^{15}$ This was a naturalistic study of individuals who had enrolled on a 6-week inpatient intensive treatment programme (ITP) for PTSD offered by Combat Stress to UK veterans between late 2012 and early 2014. The ITP is a residential programme and consists of a mixture of individual TF-CBT and groups scheduled on weekdays from 9:00 to 17:00 that are standardised and manualised to ensure a homogenous treatment experience for participants. Individuals were assigned to a closed group of eight and are offered a minimum of 15 individual TF-CBT therapy sessions (lasting $90 \mathrm{~min}$ ) and 55 group sessions each lasting $1 \mathrm{~h}$. Individual TF-CBT was offered by psychologists and CBT therapists and focused on working on trauma memories connected to military service. Group sessions were facilitated by a multidisciplinary team consisting of psychologists, CBT therapists, occupational therapists and art therapists. Group sessions included psychoeducational groups (eg, understanding PTSD, CBT education, understanding medication, exploring the links between PTSD and memory, sleep hygiene and relaxation techniques) and symptom management groups (eg, managing anxiety, managing anger, behavioural activation for depression and mindfulness groups). In addition, occupational therapists facilitated a number of groups aimed at supporting well-being (eg, groups to support resilience, develop goal planning skills and practical groups to encourage individuals to engage in meaningful activities) and six art therapy groups were offered (once a week throughout the ITP). On a typical day participants attended two group sessions that lasted an hour each and were invited to practice newly acquired skills in between sessions. Meal times were fixed throughout the day. Over the course of the week participants attended three $90 \mathrm{~min}$ individual TF-CBT sessions.

\section{Participants}

When individuals are referred into Combat Stress they receive an initial assessment which includes a range of health measures. Individuals who screen positive for PTSD are then given a referral for an ITP assessment. Prior to admission for the ITP, individuals are assessed separately by a psychiatrist and a psychologist to explore diagnosis, comorbidity and suitability for the programme. Inclusion criteria included having a primary diagnosis of PTSD, exposure to two or more traumatic events connected to an individuals' military career and being a veteran of the UK military. In addition, where participants were on psychiatric medications, these had to be stable prior to enrolling on the ITP and participants had to remain on the same dose and medication for the duration of the ITP. Exclusion criteria included being actively suicidal, being actively dependent on alcohol, having a diagnosis of a personality disorder, being actively psychotic or whether there was evidence of a brain injury that impacts significantly on cognitive functioning. This did not exclude individuals with mild 
or moderate brain injuries. When veterans met exclusion criteria, where possible they were given appropriate support and then re-assessed at a later date. For example, this included referrals to substance misuse services or psychiatric support for active symptoms of psychosis. In addition, individuals were required to not drink alcohol or use illicit drugs during the 6-week programme.

A total of 246 participants enrolled on the ITP between 2012 and early 2014. Individuals had to stay for at least 5 weeks of the 6-week programme and attend a minimum of 15 individual TF-CBT sessions to be considered a completer of the programme. Fifteen (6\%) participants were classified as non-completers. Of these, five individuals had been asked to leave the ITP because they consumed alcohol during their stay, six individuals were deemed unsuitable for therapy by the clinical team, three individuals had to leave early because of complicated physical health difficulties that arose during their admission, and one individual had to leave early because a family member became unwell.

A total of 231 individuals completed the ITP and 186 $(81 \%)$ of these were successfully followed up at 6 months. Of the 45 non-responders (19\%), 22 were contacted for a non-responder study, one had died (of natural causes), eight had withdrawn their consent to be contacted for follow-up and it was not possible to contact the remaining 14 . This means that $49 \%$ of our non-responders were contacted for the non-responder study. This increased to $65 \%$ when individuals who had died or refused further contact were removed. An overview of the sample is provided in figure 1 .

\section{Outcome measures}

Outcome measures were collected at admission, discharge, 6 weeks and at 6 months follow-up. At each of these time points the treating clinician completed a 'clinician' pack of measures and participants were asked to complete a 'veterans' pack of measures. Our primary outcomes for this study were measures of mental health difficulties and our secondary outcomes were measures of functional impairment.

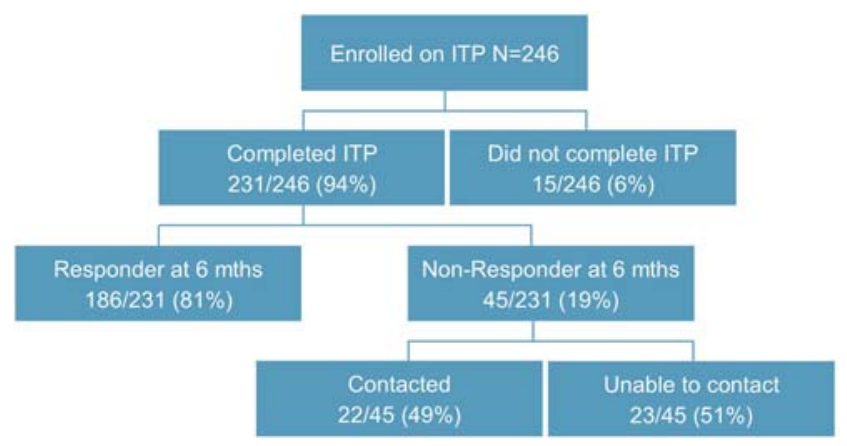

Figure 1 Overview of participants.

\section{Primary outcome measures}

The clinician completed the PTSD Symptom Scale Interview (PSS-I). The PSS-I is a 17-item semistructured interview that assesses the presence and severity of diagnostic symptoms of PTSD using the Diagnostic and Statistical Manual Fourth Edition (DSM-IV). ${ }^{16}{ }^{17}$ In addition, the participants were asked to complete a number of measures. These included the Revised Impact of Events Scale (IES-R) which measures symptoms of PTSD with 22-items. ${ }^{18}$ To explore other mental health difficulties participants completed the Patient Health Questionnaire (PHQ-9) which explores symptoms of depression; ${ }^{19}{ }^{20}$ the Generalised Anxiety Disorder Assessment (GAD-7) which explores symptoms of anxiety; ${ }^{21}$ and the Dimensions of Anger Reactions (DAR-5) to measure symptoms of anger $^{22}$

\section{Secondary outcome measures}

Clinicians completed the Global Assessment of Function (GAF). This is a numeric scale from 1 to 100 that is used to subjectively rate social, occupational and psychological functioning. The GAF is described in the DSM-IV. ${ }^{17}$ Clinicians also completed the Health of the Nation Outcome Scales (HONOS); this has 12 items and measures behaviours, impairment, symptoms and social functioning. ${ }^{23}$ Participants completed the Work and Social Adjustment Scale (WSAS). The WSAS is a self-report questionnaire that measures an individuals' perspective on their level of impaired functioning. ${ }^{24}$

\section{Demographic characteristics}

Demographic details were collected on all participants, with additional information about service (Royal Navy, Army or RAF), last rank (Officer or other rank), year they left, number of deployments they went on and how they left the military.

\section{Non-responder study measures}

Those who failed to respond at 6 months were contacted and invited to participate in a non-responder study. Researchers attempted to telephone non-responders three times to ask them to complete a number of health measures. These were the PSS-I to measure symptoms of PTSD and the PHQ-9 and GAD-7 to measure comorbid symptoms of depression and anxiety respectively.

\section{Analysis}

The first stage was to conduct exploratory analyses to assess whether potential biases were presented. To do this we used Mann-Whitney $U$ tests to explore whether differences in baseline health scores were present between individuals who completed the ITP and individuals who did not. Following this we used $\chi^{2}$ tests to explore sociodemographic differences between responders and non-responders. As detailed above, a nonresponder study was conducted which collected health measures from individuals we were able to contact. 
Mann-Whitney U tests were used to compare the health scores between responders and non-responders.

The final stage of the analysis was to explore our primary and secondary outcomes following treatment. Random slopes non-linear growth models were fitted to explore the longitudinal health and functional impairment data collected at admission, discharge, 6 weeks and 6 months follow-up. ${ }^{25}$ These analyses were repeated and adjusted for age group $(<35,35-44$ and $>45)$ and employment status. This is because these were found to improve the fit of the models using likelihood ratio tests. The models fitted were non-linear and used a fixed coefficient of time squared. Analyses were conducted using Stata V.13 (StataCorp, College Station, Texas, USA).

\section{RESULTS}

Table 1 describes the sociodemographic characteristics of the entire sample. The majority were men, had served in the Army and were in the lower ranks. Significant periods of time had passed been participants leaving the Forces and engaging in treatment with Combat Stress. Over a third of the sample stated that they had had at least a 25 year interval between leaving the military and engaging for treatment with Combat Stress. The mean number of years between leaving the military and enrolling on the ITP was 14.6 years $(95 \%$ CI 13.2 to 16.0). $72 \%$ of the sample described how this was the first time they had received psychological treatment for PTSD, although we were unable to validate this without access to lifetime medical records. This is higher than we predicted, but similar to that reported from studies of UK and US military personnel which asked participants with mental health difficulties whether they had been able to access support for their difficulties. ${ }^{26} 27$ In these studies, $77 \%$ of UK military personnel and $75 \%$ of US personnel with mental health difficulties reported that they had not accessed support. Stigma has been highlighted as the most significant barrier to help seeking. ${ }^{10}$ The majority of individuals reported having deployed to potentially hostile zones during their military careers with over $40 \%$ having deployed to three or more conflict zones. Fifty-five per cent of the sample had deployed to peace keeping operations in Northern Ireland, 33\% to Iraq since 2003, 17\% to Afghanistan since 2002 and $16 \%$ of the sample to the Falkland's war.

Table 2 demonstrated that no significant health differences were observed between individuals who completed the ITP and those who did not. Table 3 reported on the differences between individuals who responded at 6 months follow-up and those who did not. No significant differences in sociodemographic characteristics or admission health differences were observed between those who responded at 6 months and those who did not. Comparison was made between the 6 months health outcomes of responders and the 22 nonresponders where health data had been collected. No significant health differences were present between

\begin{tabular}{|c|c|}
\hline Variable & Number* (\%) \\
\hline Total enrolled on ITP & 246 \\
\hline \multicolumn{2}{|l|}{ Age group } \\
\hline$<35$ & $61(25)$ \\
\hline $35-44$ & $84(35)$ \\
\hline$>45$ & $97(40)$ \\
\hline \multicolumn{2}{|l|}{ Sex } \\
\hline Male & $241(98)$ \\
\hline Female & $5(2)$ \\
\hline \multicolumn{2}{|l|}{ Education } \\
\hline Low (O Levels or none) & $223(93)$ \\
\hline High (A Levels or above) & $17(7)$ \\
\hline \multicolumn{2}{|l|}{ Relationship status } \\
\hline In a relationship & $130(53)$ \\
\hline Single & $113(47)$ \\
\hline \multicolumn{2}{|l|}{ Employment status } \\
\hline Working & $90(39)$ \\
\hline Not working & $143(62)$ \\
\hline \multicolumn{2}{|l|}{ Financial difficulties } \\
\hline No & $113(48)$ \\
\hline Yes & $120(52)$ \\
\hline \multicolumn{2}{|l|}{ Service } \\
\hline Royal Navy & $27(11)$ \\
\hline Army & $204(84)$ \\
\hline Royal Air Force & $11(5)$ \\
\hline \multicolumn{2}{|l|}{ Role in military } \\
\hline Non-combat & $56(23)$ \\
\hline Combat & $186(77)$ \\
\hline \multicolumn{2}{|l|}{ Last rank } \\
\hline Officer & $14(6)$ \\
\hline Other ranks & $228(94)$ \\
\hline \multicolumn{2}{|c|}{ Type of discharge from military } \\
\hline Voluntary & $144(61)$ \\
\hline Non-voluntary & $91(39)$ \\
\hline \multicolumn{2}{|l|}{ Years since left the military } \\
\hline $1-9$ & $97(40)$ \\
\hline $10-19$ & $66(27)$ \\
\hline $20-29$ & $60(25)$ \\
\hline $30+$ & $20(8)$ \\
\hline \multicolumn{2}{|c|}{ Number of deployments to conflicts zones } \\
\hline 1 or 2 & $139(57)$ \\
\hline $3+$ & $103(43)$ \\
\hline
\end{tabular}

responders and non-responders. Overall, we did not find evidence of responder bias on our key variables.

The final stage of the analysis was to explore the longitudinal primary health outcomes (see table 4). Significant score reductions on both measures of PTSD were observed indicating a reduction in PTSD symptoms. In a separate analysis it was observed that $13 \%$ of participants PSSI scores remained the same or got worse, 27\% scores reduced between 1 and 9 points, $30 \%$ between 10 and 19 points and $30 \%$ by more than 20 points. At admission $100 \%$ of participants met case criteria for PTSD on the PSS-I and IES-R. At 6 weeks follow-up this reduced to $59 \%$ met case criteria for PTSD on the PSS-I and $63 \%$ on the IES-R. These rates 
Table 2 Comparisons of baseline mental health scores between completers and non-completers of the ITP

\begin{tabular}{llll}
\hline & $\begin{array}{l}\text { Completers } \\
\text { score (95\% Cl) }\end{array}$ & $\begin{array}{l}\text { Non-Completers } \\
\text { score }(95 \% \text { Cl) }\end{array}$ & p Value \\
\hline PSS-I & $36.4(35.5$ to 37.4$)$ & $35.8(32.7$ to 38.9$)$ & 0.41 \\
IES-R & $56.1(54.2$ to 58.0$)$ & $58.5(50.9$ to 66.0$)$ & 0.69 \\
PHQ-9 & $17.3(16.6$ to 17.9$)$ & $18.0(14.8$ to 21.3$)$ & 0.58 \\
GAD-7 & $15.4(14.8$ to 16.1$)$ & $16.5(14.0$ to 19.0$)$ & 0.42 \\
DAR-5 & $11.3(10.6$ to 11.2$)$ & $12.6(8.78$ to 16.4$)$ & 0.29 \\
\hline DAR-5, Dimensions of Anger Reactions; GAD-7, Generalised \\
Anxiety Disorder Assessment; IES-R, Revised Impact of Events \\
Scale; ITP, intensive treatment programme; PHQ-9, Patient Health \\
Questionnaire; PSS-I, PTSD Symptom Scale Interview.
\end{tabular}

remain roughly consistent at 6 months follow-up with $62 \%$ and $64 \%$ meeting case criteria on the PSS-I and IER-S, respectively. We then explored the other measures of mental health difficulties. Reductions in scores for the measures of depression (PHQ-9), anxiety (GAD-7) and anger (DAR-5) were observed. Overall, these reductions appeared to be more modest than for PTSD symptoms. The final data presented is of our secondary measures of functional impairment. Both the clinician completed (GAF and HONOS) measures showed significant improvement and that these gains were still present at 6 months follow-up (higher GAF scores indicate better functioning). This is supported by the self-reported measure of functioning (WSAS).

\section{DISCUSSION}

In this study we observed that veterans with PTSD who completed a 6-week treatment programme reported statistically significant improvements in their PTSD symptoms, our primary outcome. This improvement was strongest at the end of therapy and we also found evidence of a continuing reduction at follow-up time points. Crucially we found that these gains were maintained at 6 months follow-up. We observed significant reductions in depression, anxiety and problems with anger scores that were also maintained at follow-up, but these were more modest than for symptoms of PTSD. Significant improvements in functioning across a range of other secondary outcomes were observed which were maintained at follow-up. This improvement was evident in clinical functioning and also work and social

Table 3 Comparison of sociodemographics characteristics and health outcomes between responders at 6 months and non-responders

\begin{tabular}{|c|c|c|c|}
\hline & $\begin{array}{l}\text { Responder } \\
\text { N (\%) } \\
186(81) \\
\end{array}$ & $\begin{array}{l}\text { Non-responder } \\
\text { N (\%) } \\
45(19)\end{array}$ & p Value \\
\hline \multicolumn{4}{|l|}{ Age group } \\
\hline$<35$ & $44(24)$ & $14(31)$ & \\
\hline $35-44$ & $64(35)$ & $14(31)$ & \\
\hline$>45$ & $76(41)$ & $17(38)$ & 0.61 \\
\hline \multicolumn{4}{|l|}{ Sex } \\
\hline Male & $182(98)$ & $44(98)$ & \\
\hline Female & $4(2)$ & $1(2)$ & 0.98 \\
\hline \multicolumn{4}{|l|}{ Education } \\
\hline Low (O Levels or none) & $168(92)$ & $44(98)$ & \\
\hline $\begin{array}{l}\text { High } \\
\text { (A Levels or above) }\end{array}$ & $14(8)$ & $1(2)$ & 0.19 \\
\hline \multicolumn{4}{|l|}{ Relationship status } \\
\hline In a relationship & $96(52)$ & $24(53)$ & \\
\hline Single & $89(48)$ & $21(47)$ & 0.87 \\
\hline \multicolumn{4}{|l|}{ Employment status } \\
\hline Working & $66(37)$ & $19(44)$ & \\
\hline Not working & $111(63)$ & $24(56)$ & 0.41 \\
\hline \multicolumn{4}{|l|}{ Financial difficulties } \\
\hline No & $88(50)$ & $21(48)$ & \\
\hline Yes & $89(50)$ & $23(52)$ & 0.81 \\
\hline Health outcomes at admission & $\mathrm{N}=186$ & $\mathrm{~N}=45$ & \\
\hline PSS-I & 36.8 (37.7-37.9) & $34.9(32.8-37.0)$ & 0.14 \\
\hline PHQ-9 & $17.3(16.6-18.1)$ & $17.0(15.5-18.6)$ & 0.75 \\
\hline GAD-7 & $15.4(14.7-16.0)$ & $15.8(13.5-18.0)$ & 0.96 \\
\hline Health outcomes at F/U & $\mathrm{N}=186$ & $\mathrm{~N}=22$ & \\
\hline PSS-I & $23.8(22.1-25.6)$ & $25.9(21.3-30.5)$ & 0.40 \\
\hline PHQ-9 & $13.7(12.7-14.6)$ & $12.3(9.4-15.2)$ & 0.25 \\
\hline GAD-7 & $12.3(11.4-13.2)$ & $10.9(7.78-14.0)$ & 0.34 \\
\hline
\end{tabular}




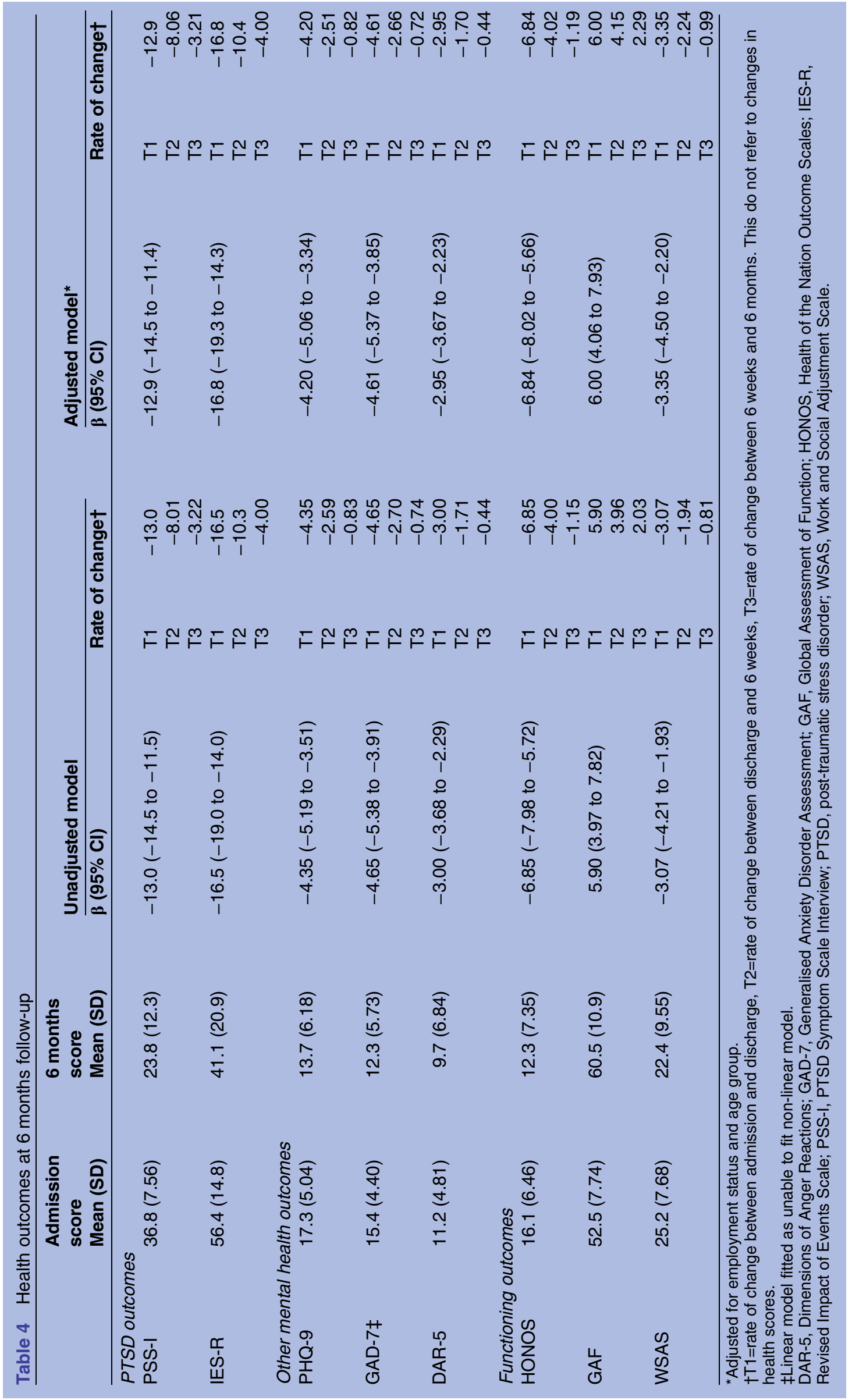


functioning. While we were unable to measure the length of time participants had been suffering from PTSD symptoms directly, we have used time since leaving the military as a proxy measure for when participants had been exposed to their index trauma. This is because an inclusion criterion for the study was that a participant's index trauma had to have occurred during their military service. The mean duration was 14.6 years. Given the considerable periods of time that participants could have been experiencing symptoms of PTSD this reduces the likelihood of spontaneous recovery. Eightyseven per cent of our sample had a reduction in their PTSD symptoms, this compares favourably to a recent study of US veterans that reported $49 \%$ of their sample reported improvements in PTSD symptoms that were maintained at follow-up. ${ }^{28}$

Comparison of baseline and 6 months post-treatment scores demonstrated that statistically significant reductions occurred. Whether the mean post-treatment scores presented reflected changes in probable PTSD diagnosis is a different matter. For example, a score of 33 and above has been used a cut-off for probable PTSD using the IES-R. Based on our findings the mean posttreatment score would still be above this cut-off. However, this cut-off has not been validated within this population which makes the interpretation difficult. A team that explored cut-offs for meeting case criteria on the IES-R within Australian Vietnam War veterans did report that a cut-off of 33 is appropriate, however the authors of this report also acknowledged they were unable to validate the cut-off within veterans seeking treatment, but rather within a community sample which limits generalisbility to this sample. ${ }^{29}$ In addition, cultural differences exist between Australian Vietnam War veterans and current UK veterans. The authors went on to comment on the limited use of the IES-R as a diagnostic tool within this population. ${ }^{29}$ However, cautious interpretation of our results is needed because while they demonstrate significant reductions in PTSD scores post-treatment they do not provide evidence that participants no longer met criteria for probable PTSD.

In view of these findings we conclude that offering individuals a mixture of group sessions aimed at emotional regulation and individual TF-CBT appears to be an effective intervention for reducing the severity of PTSD symptoms within a veteran population. Further, our findings support previous research that indicates offering TF-CBT intensively with multiple sessions per week is as effective as weekly sessions delivered over a longer period of time. ${ }^{30} 31$

The pattern of the demographic characteristics reported within the current study is similar to previously published findings from a representative cohort study of the UK Armed Forces, which suggests that certain sociodemographic groups are at higher risk of chronic presentations of PTSD. ${ }^{1}$ In particular, members of the Army, males, individuals who had combat roles and other ranks appear to be over-represented within the current study. Alternatively, this could also suggest that certain groups find it easier to engage in clinical services than others, however, given the similarity with previously published findings this appears unlikely. The noncompletion of treatment rate reported within the current study appears to be substantially lower than rates of between $22 \%$ and $46 \%$ noted in previous research of treatment non-completion for mental health interventions within the wider general public ${ }^{32-34}$ and treatment dropout rates of $21.5 \%$ in US veterans engaged in an intervention for PTSD. ${ }^{28}$ The reasons for this disparity between rates are unknown. Combat Stress operates a community outreach assessment to determine eligibility before veterans are offered a place on the programme, which may have played a part in reducing the rate of drop out.

Our findings are similar to those reported in Canadian and Australian veterans where significant improvements in terms of a reduction in PTSD symptoms and improved functioning that were maintained at follow-up have been observed. ${ }^{55}$ What appears to be central to these programmes was offering a combination of both group and individual therapy. In contrast, a recent study of US veterans with PTSD where the intervention consisted solely of group sessions found initial gains at the end of therapy but then a resurgence of PTSD symptoms at follow-up. ${ }^{28}$ This is mirrored by other older studies of US veterans involved in treatment programmes that consisted primarily of group sessions. ${ }^{4} 3637$ Further support for the inclusion of individual trauma therapy comes from a meta-analysis of treatment for PTSD provided to US veterans by the Veterans Affairs agency, which demonstrated that interventions that included exposure to trauma memories showed more promising results than interventions without. ${ }^{14}$

Meta-analyses have shown that psychological interventions for PTSD are less effective for veterans than for other groups of patients with PTSD. ${ }^{11}{ }^{12}$ This has also been demonstrated in meta-analyses of pharmacological treatments for PTSD with veterans. ${ }^{38} 39$ The reasons for this are not known, but it could be related to either the nature of military trauma, or something particular about the characteristics of veteran populations as they tend to consist of young men from deprived backgrounds who present with a range of comorbidities, severe functional impairment and high rates of substance misuse. ${ }^{40} \mathrm{We}$ have shown that offering a treatment adapted specifically for the needs of veterans, to include a combination of group sessions and individual therapy and offered within a dedicated veteran clinics rather than general clinics, may be an effective intervention for ex-military personnel with PTSD.

\section{Strengths and weaknesses}

Strengths of our study were that we used a naturalistic design, collected data from a clinical population and that the ITP has been manualised, meaning that there should have been a high level of treatment fidelity. We 
found no evidence of either a non-completer or nonresponder health bias which could have limited our findings. We believe that our sample should be representative of the wider veteran population seeking support for PTSD, as Combat Stress is the largest provider of PTSD treatment for veterans in the $\mathrm{UK}^{41}{ }^{42}$ and that there was similarity in terms of demographic characteristics between the sample described in the current study and previous epidemiological research describing the characteristics of UK military personnel with selfreported symptoms of PTSD. ${ }^{1}$ A further strength was that we used a broad-base of assessment tools. Our exclusion criterion meant that certain PTSD subgroups were not represented in our sample; in particular, individuals who meet both diagnostic criteria for having a personality disorder as well as PTSD. Further, women were under-represented when compared to the wider military population. An obvious weakness is that we did not carry out an RCT. This was because we were commissioned by the NHS to provide an intervention for veterans with PTSD based on previous programmes run in Australia that had already been shown to be effective. ${ }^{3}$ We have taken this opportunity to evaluate the ITP but accept all the limitations of a non-random comparison. We were unable to collect information related to the psychiatric medication that participants could have been taking. Given that participants' medication regimes had to be stable prior to enrolling on the ITP this suggests that gains following the ITP may have resulted from the programme. As mentioned previously, we believe that psychiatric support offered to participants prior to them engaging on the ITP was an important part of treatment to manage comorbid mental health difficulties, and may have acted as an adjuvant for the psychological intervention, as noted previously within Canadian veterans. ${ }^{43}$

\section{CONCLUSIONS}

Overall, we provide evidence to suggest that intensive treatment programmes that combine psychoeducational and emotional regulation therapy groups with individual TF-CBT reduce the burden of symptoms in veterans presenting with PTSD, comorbid mental health difficulties and severe functional impairment. Our findings are encouraging and support previous work that found that having veteran specific clinics improved engagement and treatment outcomes. However, it would be prudent for an RCT to be conducted in the future to formally test the efficacy of the intervention described within this study.

Contributors DM was involved in the design of the study, developed the analytical strategy for the paper, conducted the analyses and wrote the paper. GH was involved in the design of the study, data collection and commented extensively on the paper. CC was involved in the design of the study, data collection and commented on the paper. LS-H was involved in the design of the study, data collection and commented on the paper. MH developed the design for the study. SW was involved in the design of the study and commented extensively on the paper. WB is the chief investigator for the study, was involved in developing the analytic strategy and commented extensively on the paper.

Funding This research received no specific grant from any funding agency in the public, commercial or not-for-profit sectors.

Competing interests SW is a trustee of Combat Stress.

Ethics approval Ethical approval for this study was granted by the Combat Stress Ethics Committee.

Provenance and peer review Not commissioned; externally peer reviewed.

Data sharing statement No additional data are available.

Open Access This is an Open Access article distributed in accordance with the Creative Commons Attribution Non Commercial (CC BY-NC 4.0) license, which permits others to distribute, remix, adapt, build upon this work noncommercially, and license their derivative works on different terms, provided the original work is properly cited and the use is non-commercial. See: http:// creativecommons.org/licenses/by-nc/4.0/

\section{REFERENCES}

1. Fear NT, Jones M, Murphy D, et al. What are the consequences of deployment to Iraq and Afghanistan on the mental health of the UK armed forces? A cohort study. Lancet 2010;375:1783-97.

2. NICE. The management of PTSD in adults and children in primary and secondary care. London: National Institute for Health and Care Excellence, 2005.

3. Forbes D, Lewis V, Parslow R, et al. Naturalistic comparison of models of programmatic interventions for combat-related post-traumatic stress disorder. Aust N Z J Psychiatry 2008:42:1051-9.

4. Johnson $D$, Rosenheck R, Fontana A, et al. Outcome of intensive inpatient treatment for combat-related posttraumatic stress disorder. Am J Psychiatry 1996;153:771-7.

5. Richardson D, Eihai J, Sareen J. Predictors of treatment response in Canadian combat and peacekeeping veterans with military-related PTSD. J Nerv Ment Dis 2011;199:639-45.

6. Richardson D, Contractor A, Armour C, et al. Predictors of long-term treatment outcome in combat and peacekeeping veterans with military-related PTSD. J Clin Psychiatry 2014;75:1299-305.

7. Baum N, Brom D, Pat-Horenczyk R, et al. Transitioning from the battlefield to home: an innovative program for soldiers. J Aggres Maltreat Trauma 2013;22:644-59.

8. Resick P, Monson C, Chard K. Cognitive processing therapy: veteran/military version. Washington, DC: Department of Veterans' Affairs, 2007.

9. Murphy D, Hunt E, Luzon O, et al. Exploring positive pathways to care for members of the UK Armed Forces receiving treatment for PTSD: a qualitative study. Eur J Psychotraumatol 2014;5.

10. Iversen AC, van Staden $\mathrm{L}$, Hughes $\mathrm{JH}$, et al. The stigma of mental health problems and other barriers to care in the UK Armed Forces. BMC Health Serv Res 2011;11:31.

11. Bisson J, Ehlers A, Matthews R, et al. Psychological treatments for chronic post-traumatic stress disorder: systematic review and meta-analysis. Br J Psychiatry 2007;190:97-104.

12. Bradley R, Greene J, Russ E, et al. A multi-dimensional meta-analysis of psychotherapy for PTSD. Am J Psychiatry 2005;162:214-27.

13. Sheffield University. Veterans' Pilot Clinical Services Evaluation. University of Sheffield, 2010.

14. Goodson J, Helstrom A. Treatment of posttraumatic stress disorder in US combat veterans: a meta-analytic review. Psychol Rep 2011;109:573-99.

15. Dandeker C, Iversen A, Ross J, et al. What is a veteran? Armed Forces Soc 2006;32:161-77.

16. Foa EB, Riggs D, Dancu C, et al. Reliability and validity of a brief instrument for assessing post-traumatic stress disorder. J Trauma Stress 1993;6:459-73.

17. APA. Quick reference to the diagnostic criteria from DSM-IV. Washington, DC: American Psychiatric Association, 2005.

18. Weiss $D$, Marmar $C$. The impact of event scale-revised. In: Wilson J, Keane TM, eds. Assessing psychological trauma and PTSD. New York: Guilford, 1996:399-441.

19. Kroenke K, Spitzer R. The PHQ-9: a new depression diagnostic and severity measure. Psychiatr Ann 2002;32:509-15.

20. Titov N, Dear BF, McMillan D, et al. Psychometric comparison of the PHQ-9 and BDI-II for measuring response during treatment of depression. Cogn Behav Ther 2011;40:126-36. 
21. Swinson RP. The GAD-7 scale was accurate for diagnosing generalised anxiety disorder. Evid Based Med 2006;11:184.

22. Forbes D, Alkemade N, Mitchell D, et al. Utility of the Dimensions of Anger Reactions-5 (DAR-5) scale as a brief anger measure. Depress Anxiety 2014;31:166-73.

23. Wing J, Curtis R, Beevor A. HONOS: Health of the Nation Outcome Scales: Report on Research and Development July 1993-December 1995. London: Royal College of Psychiatrists, 1996

24. Mundt JC, Marks IM, Shear MK, et al. The Work and Social Adjustment Scale: a simple measure of impairment in functioning. Br J Psychiatry 2002;180:461-4.

25. Steele F. Multilevel models for longitudinal data. J R Stat Soc Ser A 2008;171:5-19.

26. Iversen AC, van Staden L, Hughes $\mathrm{JH}$, et al. Help-seeking and receipt of treatment among UK service personnel. Br J Psychiatry 2010;197:149-55.

27. Hoge CW, Auchterlonie JL, Milliken CS. Mental health problems, use of mental health services, and attrition from military service after returning from deployment to Iraq or Afghanistan. J Am Med Assoc 2006;295:1023-32

28. Currier J, Holland J, Drescher K. Residential treatment for combat-related posttraumatic stress disorder: identifying trajectories of change and predictors of treatment response. PLoS One 2014;9: e101741.

29. Creamer M, Bell R, Failla S. Psychometric properties of the Impact of Event Scale-Revised. Behav Res Ther 2003;41:1489-96.

30. Ehlers A, Clark DM, Hackmann A, et al. Intensive cognitive therapy for PTSD: a feasibility study. Behav Cogn Psychother 2010;38:383-98.

31. Ehlers A, Hackmann A, Grey N, et al. A randomized controlled trial of 7-day intensive and standard weekly cognitive therapy for PTSD and emotion-focused supportive therapy. Am J Psychiatry 2014;171:294-304.
32. Wierzbicki M, Pekarik G. A meta-analysis of psychotherapy dropout. Prof Psychology Res Pract 1993;24:190-5.

33. Olfson M, Mojtabai R, Sampson N, et al. Dropout from outpatient mental health care in the United States. Psychiatr Serv 2009;60:898-907.

34. O'Brien A, Fahmy R, Singh P. Disengagement from mental health services. Soc Psychiatry Psychiatr Epidemiol 2009;44:558-68.

35. Creamer M, Morris P, Biddle D, et al. Treatment outcome in Australian veterans with combat-related posttraumatic stress disorder: a cause for cautious optimism? J Trauma Stress 1999;12:545-58.

36. Fontana A, Rosenheck R. Effectiveness and cost of the inpatient treatment of posttraumatic stress disorder: comparison of three models of treatment. Am J Psychiatry 1997;154:758-65.

37. Hammarberg M, Silver S. Outcome for treatment for post-traumatic stress disorder in a primary care unit serving Vietnam veterans. $J$ Trauma Stress 1994;7:195-216.

38. Watts B, Schnurr P, Mayo L, et al. Meta-analysis of the efficacy of treatments for posttraumatic stress disorder. J Clin Psychiatry 2013;74:e541-550.

39. Jonas D, Cusack K, Forneris C, et al. Psychological and pharmacological treatments for adults with posttraumatic stress disorder (PTSD). Rockville, MD, USA: Agency for Healthcare Research and Quality. Comparative Effectiveness Review, 2013:92.

40. Iversen $\mathrm{AC}$, van Staden $\mathrm{L}$, Hughes $\mathrm{JH}$, et al. The prevalence of common mental disorders and PTSD in the UK military: using data from a clinical interview-based study. BMC Psychiatry 2009;9:68

41. van Hoorn L, Jones $\mathrm{N}$, Busuttil W, et al. Iraq and Afghanistan veteran presentations to Combat Stress since 2003. Occup Psychiatry 2013;63:238-41.

42. Combat Stress. Combat Stress 2013 annual review of clinical services. 2013.

43. Richardson D. Military-related PTSD in Canadian veterans. Can Psychiatry 2009;5:21-22. 